\title{
A COUPLED SINGLE CELL-POPULATION-BALANCE MODEL FOR MAMMALIAN CELL
}

\section{CULTURES}

Fabio R. Sidoli ${ }^{1,2}$, Athanasios Mantalaris ${ }^{1,2, *} \&$ Steven P. Asprey ${ }^{3}$

${ }^{1}$ Centre for Process Systems Engineering and ${ }^{2}$ Department of Chemical Engineering and Chemical

Technology, Imperial College London, South Kensington campus, London SW7 2AZ, UK.

${ }^{3}$ Citigroup, Citigroup Centre, 25-33 Canada Square, Canary Wharf, London E14 5LB, UK.

*Author to whom all correspondence should be addressed. Tel: +44 (0)20 7594 5601, Fax: +44 (0)20 7594

5604, a.mantalaris@ imperial.ac.uk.

1 Page

2 Tables 
Table 1: Nominal values and bounds used for time-varying inputs.

\begin{tabular}{llllllllll}
\hline No. & Input & $\mathrm{u}_{\mathrm{i}}^{\mathrm{L}}$ & $\mathrm{u}_{\mathrm{i}}{ }^{\mathrm{O}}$ & $\mathrm{u}_{\mathrm{i}}^{\mathrm{U}}$ & No. & Input & $\mathrm{u}_{\mathrm{i}}^{\mathrm{L}}$ & $\mathrm{u}_{\mathrm{i}}{ }^{\mathrm{O}}$ & $\mathrm{u}_{\mathrm{i}}^{\mathrm{U}}$ \\
\hline 1 & Feed flow rate & 0 & $2.000 \mathrm{E}-02$ & $2.771 \mathrm{E}-02$ & 15 & Phenylalanine & 0 & $4.484 \mathrm{E}+02$ & $4.484 \mathrm{E}+03$ \\
2 & Alanine & 0 & $5.008 \mathrm{E}+02$ & $5.008 \mathrm{E}+03$ & 16 & Proline & 0 & $4.736 \mathrm{E}+02$ & $4.736 \mathrm{E}+03$ \\
3 & Arginine & 0 & $5.413 \mathrm{E}+02$ & $5.413 \mathrm{E}+03$ & 17 & Serine & 0 & $4.880 \mathrm{E}+02$ & $4.880 \mathrm{E}+03$ \\
4 & Asparaginine & 0 & $5.259 \mathrm{E}+02$ & $5.259 \mathrm{E}+03$ & 18 & Threonine & 0 & $9.491 \mathrm{E}+02$ & $9.491 \mathrm{E}+03$ \\
5 & Aspartate & 0 & $5.404 \mathrm{E}+02$ & $5.404 \mathrm{E}+03$ & 19 & Tryptophan & 0 & $8.639 \mathrm{E}+01$ & $8.640 \mathrm{E}+02$ \\
6 & Cystine & 0 & $4.699 \mathrm{E}+02$ & $4.699 \mathrm{E}+03$ & 20 & Tyrosine & 0 & $4.440 \mathrm{E}+02$ & $4.440 \mathrm{E}+03$ \\
7 & Glutamate & 0 & $5.263 \mathrm{E}+02$ & $5.262 \mathrm{E}+03$ & 21 & Valine & 0 & $9.482 \mathrm{E}+02$ & $9.482 \mathrm{E}+03$ \\
8 & Glutamine & 0 & $4.558 \mathrm{E}+03$ & $4.558 \mathrm{E}+04$ & 22 & Palmatic Acid & 0 & $3.000 \mathrm{E}+02$ & $3.000 \mathrm{E}+03$ \\
9 & Glycine & 0 & $5.259 \mathrm{E}+02$ & $5.259 \mathrm{E}+03$ & 23 & Ammonia & 0 & $5.000 \mathrm{E}+02$ & $5.000 \mathrm{E}+03$ \\
10 & Histidine & 0 & $3.085 \mathrm{E}+02$ & $3.085 \mathrm{E}+03$ & 24 & Glucose & 0 & $2.500 \mathrm{E}+04$ & $2.500 \mathrm{E}+05$ \\
11 & Isoleucine & 0 & $9.279 \mathrm{E}+02$ & $9.279 \mathrm{E}+03$ & 25 & Maltose & 0 & $1.000 \mathrm{E}+03$ & $5.000 \mathrm{E}+03$ \\
12 & Leucine & 0 & $9.279 \mathrm{E}+02$ & $9.279 \mathrm{E}+03$ & 26 & Pyruvate & 0 & $1.000 \mathrm{E}+03$ & $5.000 \mathrm{E}+03$ \\
13 & Lysine & 0 & $1.148 \mathrm{E}+03$ & $1.148 \mathrm{E}+04$ & 27 & Lactate & 0 & $1.500 \mathrm{E}+03$ & $1.500 \mathrm{E}+04$ \\
14 & Methioine & 0 & $2.287 \mathrm{E}+02$ & $2.287 \mathrm{E}+03$ & & & & & \\
\hline
\end{tabular}

Table 2: Nominal values and bounds used for time-invariant inputs.

\begin{tabular}{llllllllll}
\hline No. & Input & $\omega_{\mathrm{i}}^{\mathrm{L}}$ & $\omega_{\mathrm{i}}^{0}$ & $\omega_{\mathrm{i}}^{\mathrm{U}}$ & No. & Input & $\omega_{\mathrm{i}}^{\mathrm{L}}$ & $\omega_{\mathrm{i}}^{0}$ & $\omega_{i}^{\mathrm{U}}$ \\
\hline 1 & & & & & 17 & Lysine & 0 & $1.148 \mathrm{E}+03$ & $3.444 \mathrm{E}+03$ \\
2 & & & & 18 & Methionine & 0 & $2.287 \mathrm{E}+02$ & $6.860 \mathrm{E}+02$ \\
3 & Palmatic & $0 \mu \mathrm{g} / \mathrm{L}$ & $1.000 \mathrm{E}+02$ & $9.000 \mathrm{E}+02$ & 19 & Phenylalanine & 0 & $4.484 \mathrm{E}+02$ & $1.345 \mathrm{E}+03$ \\
4 & Maltose & 0 & $1.000 \mathrm{E}+02$ & $5.000 \mathrm{E}+02$ & 20 & Proline & 0 & $4.736 \mathrm{E}+02$ & $1.421 \mathrm{E}+03$ \\
5 & Glucose & 0 & $2.500 \mathrm{E}+04$ & $7.500 \mathrm{E}+04$ & 21 & Serine & 0 & $4.880 \mathrm{E}+02$ & $1.464 \mathrm{E}+03$ \\
6 & Alanine & 0 & $5.008 \mathrm{E}+02$ & $1.503 \mathrm{E}+03$ & 22 & Threonine & 0 & $9.491 \mathrm{E}+02$ & $2.847 \mathrm{E}+03$ \\
7 & Arginine & 0 & $5.413 \mathrm{E}+02$ & $1.624 \mathrm{E}+03$ & 23 & Tryptophan & 0 & $8.639 \mathrm{E}+01$ & $2.592 \mathrm{E}+02$ \\
8 & Asparagine & 0 & $5.259 \mathrm{E}+02$ & $1.578 \mathrm{E}+03$ & 24 & Tyrosine & 0 & $4.440 \mathrm{E}+02$ & $1.332 \mathrm{E}+03$ \\
9 & Aspartate & 0 & $5.404 \mathrm{E}+02$ & $1.621 \mathrm{E}+03$ & 25 & Valine & 0 & $9.482 \mathrm{E}+02$ & $2.845 \mathrm{E}+03$ \\
10 & Cystine & 0 & $4.699 \mathrm{E}+02$ & $1.410 \mathrm{E}+03$ & 26 & Pyruvate & 0 & $3.000 \mathrm{E}+02$ & $1.000 \mathrm{E}+03$ \\
11 & Glutamate & 0 & $5.263 \mathrm{E}+02$ & $1.579 \mathrm{E}+03$ & 27 & Oxygen & 0 & $1.000 \mathrm{E}+04$ & $2.400 \mathrm{E}+04$ \\
12 & Glutamine & 0 & $4.558 \mathrm{E}+03$ & $1.367 \mathrm{E}+04$ & 28 & Lactate & 0 & $1.000 \mathrm{E}+03$ & $4.500 \mathrm{E}+03$ \\
13 & Glycine & 0 & $5.259 \mathrm{E}+02$ & $1.578 \mathrm{E}+03$ & 29 & Ammonia & 0 & $5.000 \mathrm{E}+01$ & $1.500 \mathrm{E}+03$ \\
14 & Histidine & 0 & $3.085 \mathrm{E}+02$ & $9.256 \mathrm{E}+02$ & 30 & Carbon & 0 & $1.000 \mathrm{E}+00$ & $1.000 \mathrm{E}+02$ \\
15 & Isoleucine & 0 & $9.279 \mathrm{E}+02$ & $2.784 \mathrm{E}+03$ & 31 & Urea & 0 & $1.000 \mathrm{E}+02$ & $6.000 \mathrm{E}+02$ \\
16 & Leucine & 0 & $9.279 \mathrm{E}+02$ & $2.784 \mathrm{E}+03$ & 32 & Reactor & $0.45 \mathrm{~L}$ & $5.000 \mathrm{E}-01$ & $5.500 \mathrm{E}-01$ \\
\hline
\end{tabular}

